\title{
Positronium Formation with Trapped Electrons in $\boldsymbol{n}$-Alkanes
}

\author{
B. ZGARDZińsKA* AND T. GOWOREK
}

Institute of Physics, Maria Curie-Skłodowska University, pl. Marii Curie-Skłodowskiej 1, 20-031 Lublin, Poland

(Received March 17, 2010; in final form December 5, 2010)

Positronium (Ps) formation during self-irradiation by positrons was observed in $n$-alkanes of various carbon chain length. The rise of Ps intensity with time of irradiation is the result of positronium formation with trapped excess electrons. In the range $T<190 \mathrm{~K}$ the saturation value of positronium intensity changes with temperature mainly due to the change of positron mobility. With the increase of the irradiation dose the process of charging traps is slowing down and this effect is ruled mainly by interaction of excess electrons with ionization products during electron's migration to the traps.

PACS: 36.10.Dr, 36.20.Fz, 61.80.-x, 72.20.Jv, 78.70.Bj

\section{Introduction}

Positronium (Ps) formation in condensed matter consists in the capture of quasi-free electron by positron. In the case of high-energy positrons, e.g. from $\beta+$ decay, this process can be described by the blob model $[1,2]$. Positron combines with one of electrons from the cluster of ionization products ("blob") at the end of its ionization track. However, positrons being slowed down produce free electrons along their whole ionization tracks. At low temperature, some of these electrons can be trapped in shallow traps in the solid, e.g. in intermolecular cavities. When the sample is continuously irradiated by positrons, the number of trapped electrons rises with time. If the Ps binding energy is higher than the trap depth, free positron has additionally a possibility to form positronium with one of trapped electrons. Depending on the carbon chain length, the electron trap depth in alkanes can be estimated as $(1 \div 3) \mathrm{eV}[3]$, while Ps binding energy is $6.8 \mathrm{eV}$. Thus, excess electron trapping should lead to the rise of Ps intensity with irradiation time. This effect is well confirmed in the experiments with polymers $[4,5]$ and also with some crystalline media, like cyclohexane [6] or alkanes [7].

The total intensity of positronium consists of two components: one of blob origin, the other - related to electrons being picked up from the traps. This last one can be called "delayed component", as diffusing positrons need time to migrate to randomly distributed occupied traps. Other processes, related to the presence of various ionization products in the sample irradiated by positrons also influence observed intensity of positronium. Trapped electrons can be thrown out from the traps by light, visible or near infrared, which restores the "zero dose level" of Ps formation probability. Although illumination is effective in emptying the electron traps, it has little effect on other post-ionization products.

The aim of this paper was to collect more data about the interaction of positrons with excess electrons, in-

* corresponding author; e-mail:

bozena.zgardzinska@poczta.umcs.lublin.pl fluence of temperature, irradiation time etc. Fragmentary data concerning this problem were published by us earlier [8].

\section{Instrumental}

As the media where positronium formation takes place we have chosen three $n$-alkanes: undecane $\mathrm{C}_{11} \mathrm{H}_{24}$, heptadecane $\mathrm{C}_{17} \mathrm{H}_{36}$ and tetratriacontane $\mathrm{C}_{34} \mathrm{H}_{70}$ (the longest carbon chain). At low temperature all these alkanes form rigid crystals, but in the range preceding the melting point by about $10 \mathrm{~K}$ they exhibit so-called rotator phase with orientational degree of freedom about long molecular axis. The samples of $99 \%$ purity were from Sigma-Aldrich, and we used them without any further refinement. The samples filled the container with positron source $\left(0.3 \mathrm{MBq}\right.$ of $\left.{ }^{22} \mathrm{Na}\right)$ fixed in the centre. Molten samples were degassed by freeze-thaw technique in order to avoid the ortho-para conversion in dissolved molecular oxygen. The container was attached to the cold finger with lower end submerged in liquid nitrogen. The chamber volume over the sample was evacuated to about $0.5 \mathrm{~Pa}$. The sample temperature was regulated by heating coil placed just below the chamber. Two light emitting diodes placed inside the container could illuminate the sample when needed.

The positron annihilation lifetime spectra (PALS) were measured by a standard fast-slow delayed coincidence spectrometer with two scintillation heads; time resolution was about 250 ps FWHM. The collection time of one spectrum was $1 \mathrm{~h}$ (if not stated otherwise); such a spectrum contained typically $7 \times 10^{5}$ to $10^{6}$ coincident events. At that counting rate the dead time of spectrometer was negligible. The spectra were processed by the LT programme [9], assuming the appearance of three exponential components convoluted with the instrumental resolution curve. The shortest-lived component was ascribed to the decay of singlet para-Ps ( $p$-Ps), the intermediate one - to the annihilation of unbound positrons and the longest-lived one to the decay of ortho-Ps (o-Ps). This last component, easily separable from the remainder of spectrum, was assumed to be a measure of positronium formation probability. If there is no participation of positronium in chemical reactions, the fraction 
of positrons forming Ps should be equal $4 / 3$ of $o$-Ps intensity. For this reason at standard spectra processing described below the para-Ps to ortho-Ps ratio was fixed as 1:3 (the discussion how this constraint affects the results of fitting is given at the end of Sect. 3). A correction for positron absorption in the Kapton source envelope was also applied.

\section{Results and discussion}

Typical effects related to positron irradiation of the alkane sample are presented in Fig. 1 showing the time dependences of $o$-Ps intensity $I_{3}$ in heptadecane at $210 \mathrm{~K}$. In darkness, the intensity rises with the time constant of several hours and then decreases slowly due to the interaction of excess electrons with radicals and other ionization products. Time constants of these processes depend on the source activity. Switching on the LEDs reduces the intensity to the level of Ps production in the blob. When the sample is illuminated from the beginning of measurements, a small decrease of $o$-Ps intensity $I_{3}$ with time is observed, which means that the reactions with post-ionization products have also an influence on the blob processes. It should be noted that lack of accumulation of trapped electrons is observed not only in liquids, but also in the rotator phase of $n$-alkanes.

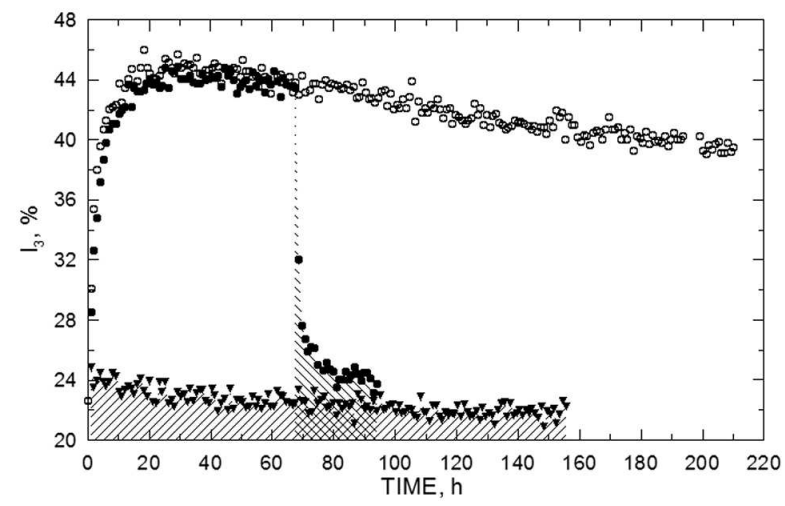

Fig. 1. Variation of ortho-positronium formation intensity $I_{3}$ with irradiation time in $n$-heptadecane $\mathrm{C}_{17} \mathrm{H}_{36}$. Open circles - in darkness, full circles $-68 \mathrm{~h}$ in darkness and next the $0.95 \mu \mathrm{m}$ LED diodes switched on, triangles - sample illuminated by LEDs from the beginning of irradiation. Temperature $210 \mathrm{~K}$.

Temperature dependence of $I_{3}$ saturation intensity was measured in $n$-heptadecane. The sample was cooled from the melt to $130 \mathrm{~K}$ and stored at that temperature for $15 \mathrm{~h}$; then temperature was increased by steps. The time of spectra collection was set for $1 \mathrm{~h}$ and the temperature was changed likewise. In heptadecane the blob intensity $I_{3}$ at $130 \mathrm{~K}$ was found to be about $20 \%$, while it reached $40 \%$ after saturation of the process of picking electrons from the traps. With the increase of temperature the $I_{3}$ value rose, too (Fig. 2), up to the maximum near $200 \mathrm{~K}$. At higher temperatures, $I_{3}$ decreased rapidly

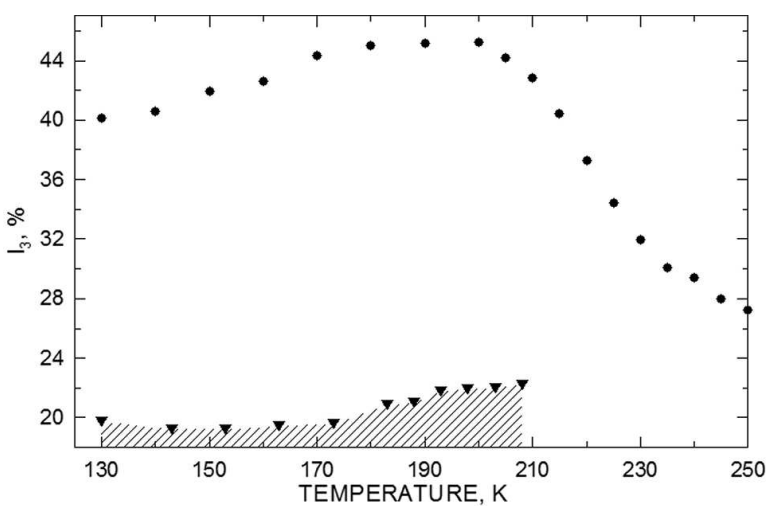

Fig. 2. Ortho-Ps intensity $I_{3}$ in $n$-heptadecane after reaching the saturation value at $130 \mathrm{~K}$, as a function of rising temperature (dots); for comparison the intensities in the sample illuminated by $0.95 \mu \mathrm{m}$ LEDs are also shown (triangles).

due to thermal collapse of the traps destroyed by activation of intra-molecular motions. The $I_{3}(T)$ dependence for tetratriacontane was almost identical.

The rise of $I_{3}$ saturation value between 130 and $200 \mathrm{~K}$ can result from either an increase of trapped electron density or greater positron mobility facilitating its migration toward the occupied traps. In order to check the mechanism of $I_{3}$ rise, the sample of heptadecane was kept for $44 \mathrm{~h}$ at the temperature of $130 \mathrm{~K}$ and then the temperature was increased to $185 \mathrm{~K}$; the sample was stored at that temperature for $11 \mathrm{~h}$ and returned stepwise to $130 \mathrm{~K}$ for $11 \mathrm{~h}$ and once more to $185 \mathrm{~K}$. The result is shown in Fig. 3. It can be seen that sudden change of temperature is immediately followed by the change of intensity up to the value corresponding to new temperature; $I_{3}$ increase is the same as seen in Fig. 2 (in that figure the temperature increase lasted $8 \mathrm{~h}$, while at the skips in Fig. 3 - less than $15 \mathrm{~min}$ ). Charging the traps occurs in the time scale of several hours (here the time constant is about $7.5 \mathrm{~h}$ ), while the intensities $I_{3}$ observed in the first spectrum after the skip were already the final ones, even after reduction of the spectrum registration time to $15 \mathrm{~min}$. It means that the transition to new $I_{3}$ values was practically immediate and during $10 \mathrm{~h}$ after the increase of temperature the intensity $I_{3}$ remained constant. The heating time was too short to allow the trapped electron density to change, thus the results indicate that the change of $I_{3}$ is due to increased mobility of positrons with temperature.

Analogous measurements were done with $n$-tetratriacontane (Fig. 4). The sample was cooled from the melt to $123 \mathrm{~K}$, stored $28 \mathrm{~h}$ at that temperature up to reaching $I_{3}$ saturation (time constant $\approx 8 \mathrm{~h}$ ) and then the cycle of steps $123-173-123 \mathrm{~K}$, like in previous case, was performed. Also in this case the intensity $I_{3}$ followed rapidly the changes of temperature. When the cycle of temperatures is repeated at slightly higher temperatures, 153-203-153 K one can notice a different behaviour of $I_{3}$ 


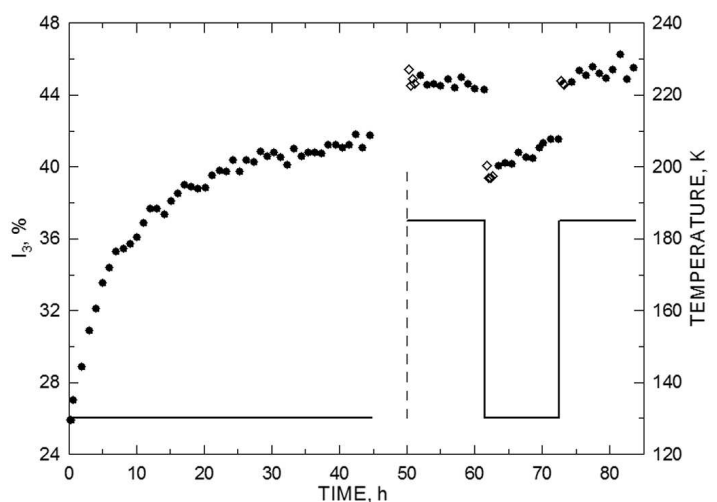

Fig. 3. Changes of intensity $I_{3}$ in $n$-heptadecane at stepwise changes of temperature (shown at the bottom of figure). Saturation of $I_{3}$ obtained after $40 \mathrm{~h}$. Measurements in darkness. The $I_{3}$ values obtained from the spectrum collection reduced to $15 \mathrm{~min}$ are shown as empty diamonds.

with time: directly after the increase of temperature up to $203 \mathrm{~K}$ the intensity $I_{3}$ is high, but decreases with time (at $173 \mathrm{~K}$ it was stable in time). It means that trapped electron density, characteristic for $153 \mathrm{~K}$, is too high for the temperature $203 \mathrm{~K}$; partial emptying of traps already begins, $I_{3}$ decreases with time constant $\approx 3 \mathrm{~h}$ to the equilibrium value. The change $\Delta I_{3}$ at the step $153-203 \mathrm{~K}$ is the same as $-\Delta I_{3}$ at reverse step. Now the trapped electron density is too low for equilibrium state and it should rise with time. It is seen in Fig. 4 that the rise is very slow. This is a common property of all alkanes we investigate here: time constant of trap loading rises with the time of irradiation. The effect is well visible in Fig. 5, where the measurements performed on a heptadecane sample at $130 \mathrm{~K}$ are shown. First $40 \mathrm{~h}$ of sample storage was the same as in Fig. 3, then after next $60 \mathrm{~h}$ the trapped electrons were removed by keeping the sample in the rotator phase, close to the melting point. The temperature was lowered again to $130 \mathrm{~K}$ and the lifetime spectra were collected during over $60 \mathrm{~h}$. In the first run the time constant of $I_{3}$ rise was about $7.5 \mathrm{~h}$, in the second one $-45 \mathrm{~h}$. Moreover, the saturation intensity $I_{3 \max }$ which in the first run was $45 \%$, in the second one the extrapolation of exponential curve fitted to the data, gives the intensity of about $37 \%$.

Trapping of excess electrons does not affect the $o$-Ps lifetime, but changes the lifetime of free positrons $\tau_{2}$. The decay rate $\lambda_{2}$ is now

$$
\lambda_{2}=\frac{1}{\tau_{2}^{0}}+K n,
$$

where $\tau_{2}^{0}$ is the lifetime in absence of trapping, $n$ the density of electrons in the traps, $K$ - the rate of positron capture by trapped electrons. Trapping leads to the shortening of free positron lifetime. One can see in Fig. 5 that the term $K n$ was smaller for the sample irradiated during over $100 \mathrm{~h}$ than in the fresh one $\left(\tau_{2}\right.$ is longer $)$.

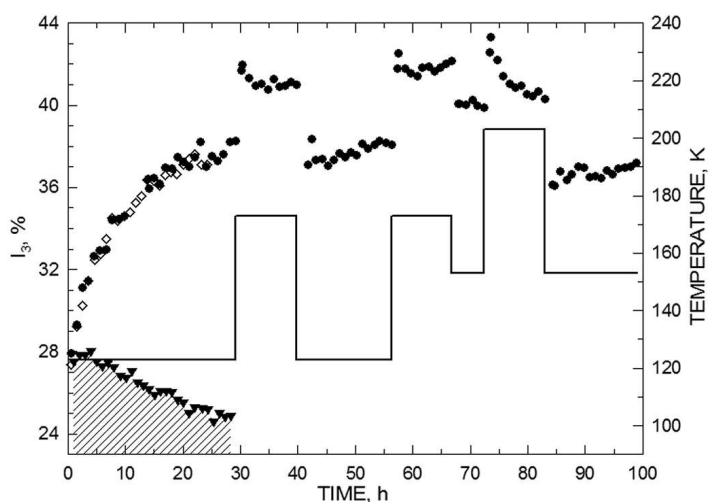

Fig. 4. Changes of intensity $I_{3}$ in $n$-tetratriacontane $\mathrm{C}_{34} \mathrm{H}_{70}$ at stepwise changes of temperature, after reaching the saturation. Solid line is the temperature graph. Triangles denote the intensity in the sample illuminated continuously; diamonds - the data obtained after melting the sample which was used in the first part of measurements.

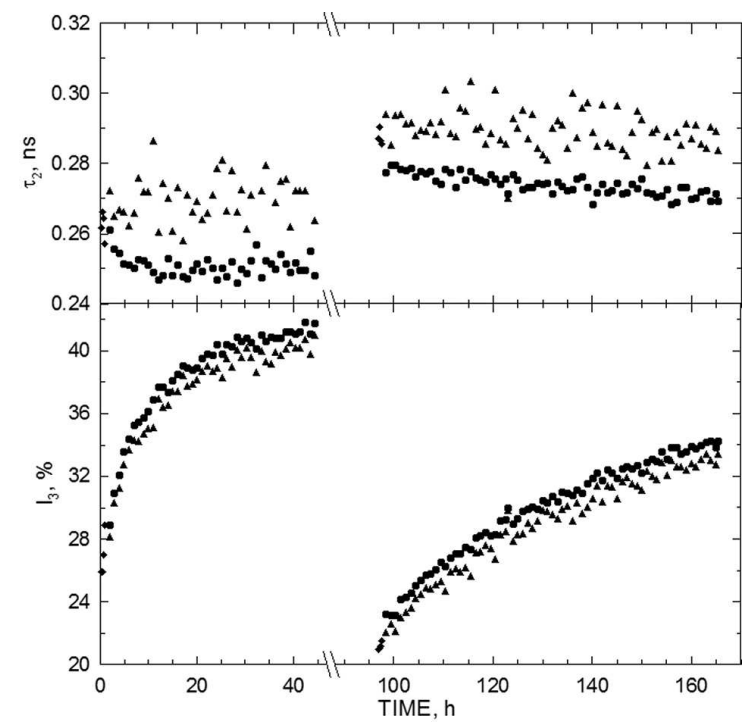

Fig. 5. Changes of $I_{3}$ intensity and free positron lifetime $\tau_{2}$ in $n$-heptadecane with time of irradiation. At $95 \mathrm{~h}$ the second run of collecting electrons in the traps begins, after preliminary emptying the traps at sufficiently high temperature $(240 \mathrm{~K})$. Full circles - spectra processed with fixed para- to ortho-Ps ratio 1:3, triangles — with fixed para-Ps lifetime. Temperature $130 \mathrm{~K}$.

There is to note that annealing the sample in the rotator phase eliminates electrons from the traps, but not the post-ionization products (radicals, ions, etc.); full restoration of $I_{3}$ dependence on time occurs after the melting (diamonds in Fig. 4).

In all data processing procedures described above we assumed the para to ortho ratio as 1:3. In order to check whether such assumption affects the results, all spectra were processed once more without any constraint. The distribution width $(2 \sigma)$ of fitted $\tau_{1}$ values (para-Ps life- 
time) was about $10 \mathrm{ps}$ and $\tau_{1}$ showed no tendency to change with temperature, thus, an average of $\tau_{1}$ was calculated (for both runs separately) and fixed. The values of $\tau_{2}$ were now slightly higher than at previous data treatment (Fig. 5), but the tendency remained unchanged in the second run the term $K n$ is smaller.

Next example of time dependence of positron lifetime spectra is shown in Fig. 6. Time variation of positron spectra for the sample of $n$-undecane $\mathrm{C}_{11} \mathrm{H}_{24}$ was observed [10]. Five cycles of electron accumulation were performed, each cycle ended with sample illumination by two near-infrared diodes $\left(\lambda_{\mathrm{m}}=0.95 \mu \mathrm{m}\right)$. After $8 \mathrm{~h}$ of illumination the electron traps were empty and the new cycle of trapping could begin. Total duration of irradiation was $350 \mathrm{~h}$. To reduce the scattering of fitted spectrum parameters the ortho- to para-Ps ratio was assumed again as 3:1. Time constant of $I_{3}$ increase, like in previous examples, lengthened with time elapse. In the first cycle it was about $10.6 \mathrm{~h}$, while in the fifth one $-32.7 \mathrm{~h}$. The $I_{3}$ value after saturation was estimated as $53 \%$ after the first cycle, and $40 \%$ after the fifth one.

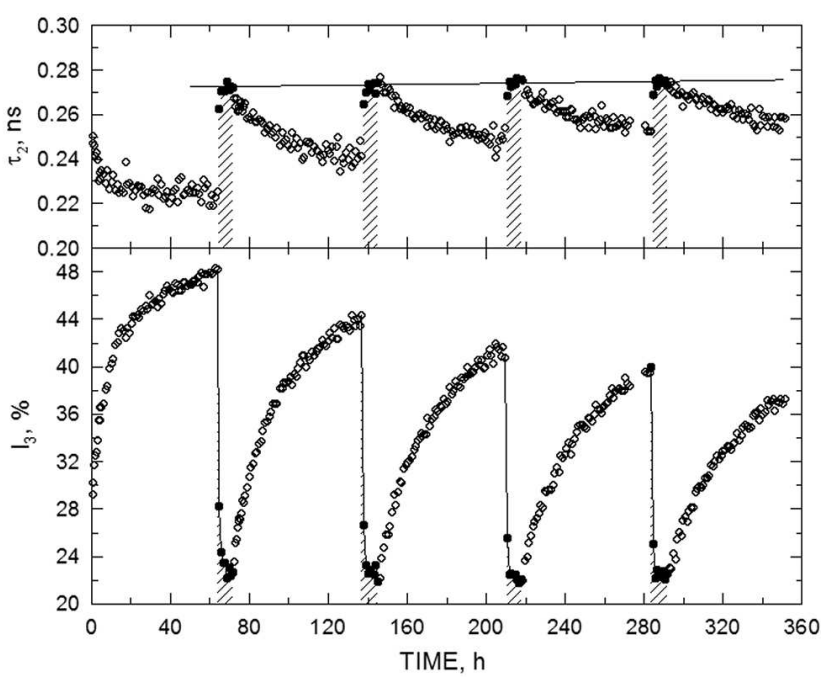

Fig. 6. Changes of $I_{3}$ intensity and free positron lifetime $\tau_{2}$ with time of irradiation in $n$-undecane $\mathrm{C}_{11} \mathrm{H}_{24}$. During the irradiation the traps were discharged four times by switching on the $0.95 \mu \mathrm{m}$ LEDs (full symbols, dashed area). Solid straight line is fitted to the final 3 points in each of four cycles of illumination. Temperature $130 \mathrm{~K}$

The lifetime $\tau_{2}$ decreased during electron accumulation and time constant of this decrease also lengthened in consecutive cycles. After illumination the $\tau_{2}$ values in each cycle returned to the same value, no change of $\tau_{2}$ was observed. Straight line fitted to the points corresponding to last $3 \mathrm{~h}$ of illumination gave the $\tau_{2}$ rise by $2.0 \mathrm{ps}$ during $200 \mathrm{~h}$, while the fitting uncertainty of individual $\tau_{2}$ point is practically the same: $\pm 1.6 \mathrm{ps}$.

\section{Conclusions}

The positronium behaviour in $n$-alkanes with various length of carbon chain (from 11 to 34 atoms) is very similar. The effect of electron trapping is best visible in the changes of $o$-Ps intensity, but also can be seen in time variations of free positron lifetime.

If the accumulation of ionization products disturbs the migration of positrons in the medium, one could expect the change of $\tau_{2}$ with time in irradiated and IR illuminated sample. Lack of such an effect can be an indication that positron capture is irradiation independent (in the limits of uncertainty). Localization of positrons, postulated by some authors [11] does not measurably modify the $\tau_{2}$ lifetime. Excess electrons produced along the ionization track are much more sensitive to the presence of irradiation products; they recombine with ions, react with radicals etc. Modification of PALS spectra with time is related mainly to the density of trapped electrons. The rise of $I_{3}$ saturation value with temperature in the range $130-190 \mathrm{~K}$ is due to increasing positron mobility; the decrease observed above $200 \mathrm{~K}$ is the result of thermal emptying the traps. In the correcting term in the expression for $\tau_{2}$ lifetime (Eq. (1)) the constant $K$ is ruled mainly by positrons, while $n$ - by trapped excess electrons.

\section{Acknowledgments}

Authors wish to thank Dr. Mark Pietrow for his participation in undecane measurements and Dr. Jan Wawryszczuk for his technical assistance.

\section{References}

[1] O.E. Mogensen, J. Chem. Phys. 60, 998 (1974).

[2] S.V. Stepanov, V.M. Byakov, J. Chem. Phys. 116, 6178 (2002).

[3] M. Pietrow, J. Wawryszczuk, Mater. Sci. Forum 666, 93 (2011).

[4] T. Suzuki, T. Miura, Y. Oki, M. Numajiri, K. Kondo, Y. Ito, J. Phys. IV 3, 283 (1993).

[5] C.L. Wang, T. Hirade, F.H.J. Maurer, M. Eldrup, N.J. Pedersen, J. Chem. Phys. 108, 4654 (1998).

[6] T. Hirade, F.H.J. Maurer, M. Eldrup, Radiat. Phys. Chem. 58, 465 (2000).

[7] N. Djourelov, T. Goworek, K. Kondo, T. Suzuki, R. Zaleski, Phys. Lett. A 323, 165 (2004).

[8] B. Zgardzińska, T. Hirade, T. Goworek, Chem. Phys. Lett. 446, 309 (2007).

[9] J. Kansy, Nucl. Instrum. Methods Phys. Res. A 374, 235 (1996).

[10] M. Pietrow, B. Zgardzińska, Acta Phys. Pol. A 110, 641 (2006).

[11] T. Hirade, N. Suzuki, F. Saito, T. Hyodo, Phys. Status Solidi C 4, 3714 (2007). 